“C 2010 IEEE. Personal use of this material is permitted. Permission from IEEE must be obtained for all other uses, in any current or future media, including reprinting/republishing this material for advertising or promotional purposes, creating new collective works, for resale or redistribution to servers or lists, or reuse of any copyrighted component of this work in other works.” 


\title{
Dynamic Multilevel Optimization of Machine Design and Control Parameters for PMSM Drive System Based on Correlation Analysis
}

\author{
Xiangjun Meng, Shuhong Wang, Jie Qiu \\ Faculty of Electrical Engineering, Xi'an Jiaotong University, Xi'an 710049, China; \\ shwang@mail.xjtu.edu.cn \\ Jian Guo Zhu, Yi Wang, Youguang Guo, Dikai Liu, Wei Xu \\ School of Electrical, Mechanical and Mechatronic Systems, University of Technology, Sydney, NSW 2007, Australia
}

\begin{abstract}
In this paper, a multilevel optimization method is proposed for a motor drive system which includes a surface mounted permanent magnet synchronous machine (SPMSM), the converter/inverter, and the control schemes. Firstly, the multilevel optimization is described by using the problem matrix which may be used to allocate the design variables on different levels. The parameters in the problem matrix are deduced by using correlation analysis. Secondly, the architecture and implementation of Multilevel Genetic Algorithm (MLGA) are carried out. As one of the advantages of MLGA, the dynamic adjustment strategy of GA operators is utilized to improve optimal performance. The algorithm is applied to a three-level optimization problem in which the optimization of SPMSM design and the control parameters of drive are considered in different levels. Finally, some results and discussions about the application of the proposed algorithm are presented.
\end{abstract}

\section{INTRODUCTION}

Multilevel optimization is an effective method to solve complex optimization problem and it has been reported. Bartheley [1] used problem matrix method to describe the relationship between the objective functions and variables. Q. $\mathrm{S}$. Li, et al. [2] presented Multilevel Genetic Algorithm (MLGA) for the optimization of actively control building under earthquake excitations. Multilevel optimizations are difficult to solve due to the characteristics of nonlinearity, multi-modal functions and mixed discrete variables.

Permanent magnetic synchronous machines (PMSMs) have been an attractive choice for many applications because of its high efficiency and power density. In this paper, MLGA is presented for design optimization of a motor drive system consisting of the drive circuit and an SPMSM controlled by using Field Oriented Control (FOC) to minimize the cost of copper and permanent magnets, and to maximize the efficiency of the motor and the drive system as well as the overshoot and ripples of output torque, speed and d-axis component of current. The finite element analysis (FEA) of the motor is used to calculate the no-load magnetic field, the backelectromagnetic force (back-EMF), the $d-$ and q-axis components of the stator winding inductances.

\section{Formulation OF MUltileVEl Optimization PROBlem}

In multilevel optimization problems, the relationship between the design variables, constraints and objective functions can be described by a Problem Matrix, as shown in
Fig.1. In Fig. 1, the symbols $\boldsymbol{P}_{x x}$, i.e. P-values, are the coefficients, which indicate the relative importance between design variables and objective functions, as well as constraints in Correlation Analysis [3]. The larger the P-value is, the less relative importance of the design variable for the objective function is. In this paper, the samples of variables are determined by Design of Experiment (DOE) method. Some commercial statistic software packages, such as Minitab, can provide the module for the relative importance analysis.

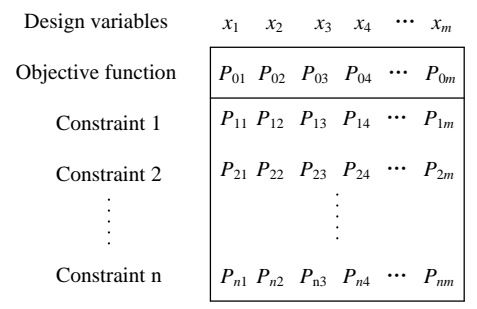

Fig. 1. Problem matrix

According to P-values in the Problem matrix, the design variables may be arranged on diverse levels. For one objective function, the variables possess similar P-values will be managed on the same level.

\section{MultileVel GenetiC Algorithm}

The architecture of MLGA is shown in Fig. 2. In MLGA the design optimization variables are classified and allocated to different levels according to the relative importance among the variables and objective functions, constraints, as well as the practical engineering weight and optimization sequence. The variables on different levels are encoded independently. Each level may have multiple populations and each of them can adopt different dynamic genetic operators and parameters. Furthermore, the relationship between sub-problems in multilevel problems can be handled by MLGA.

An independent GA can be described as follows.

$$
G A=(P O, P S, I S, F I T, S O, C O, M O)
$$

where, PO, PS, IS, FIT represent the population, the population size, the encoding length and the fitness value, respectively; $\boldsymbol{S O}, \boldsymbol{C O}, \boldsymbol{M O}$ are the genetic operations, i.e. selection, crossover and mutation.

The MLGA can be described as follows. 


$$
G A_{i j}=\left(P O_{i j}, P S_{i j}, I S_{i j}, F I T_{i j}, S O_{i j}, C O_{i j}, M O_{i j}\right)
$$

where, $G A_{i j}$ stands for applying the independent GA to the $i$ th level and the $j$ th module. In the view of the reaction between different levels and adjoint sub-modules on the same level, $G A_{i j}$ can be described as follows.

$$
\begin{aligned}
G A_{i j}= & \left(\boldsymbol{P O}_{i j}\left(G A_{i, j-1}, G A_{i-1, j}, G A_{i, j+1}\right),\right. \\
& \boldsymbol{P} \boldsymbol{S}_{i j}\left(G A_{i, j-1}, G A_{i-1, j}, G A_{i, j+1}\right), \\
& \boldsymbol{I S} \boldsymbol{S}_{i j}\left(G A_{i, j-1}, G A_{i-1, j}, G A_{i, j+1}\right), \\
& \boldsymbol{F} \boldsymbol{F T}_{i j}\left(G A_{i, j-1}, G A_{i-1, j}, G A_{i, j+1}\right), \\
& \boldsymbol{S O}_{i j}\left(G A_{i, j-1}, G A_{i-1, j}, G A_{i, j+1}\right), \\
& \boldsymbol{C} \boldsymbol{O}_{i j}\left(G A_{i, j-1}, G A_{i-1, j}, G A_{i, j+1}\right), \\
& \left.\boldsymbol{M} \boldsymbol{O}_{i j}\left(G A_{i, j-1}, G A_{i-1, j}, G A_{i, j+1}\right)\right)
\end{aligned}
$$

The $G A_{i j}$ can be affected by upper level $G A_{i-1, j}$ or same level mudles, $G A_{i, j-1}$ and $G A_{i, j-1}$.

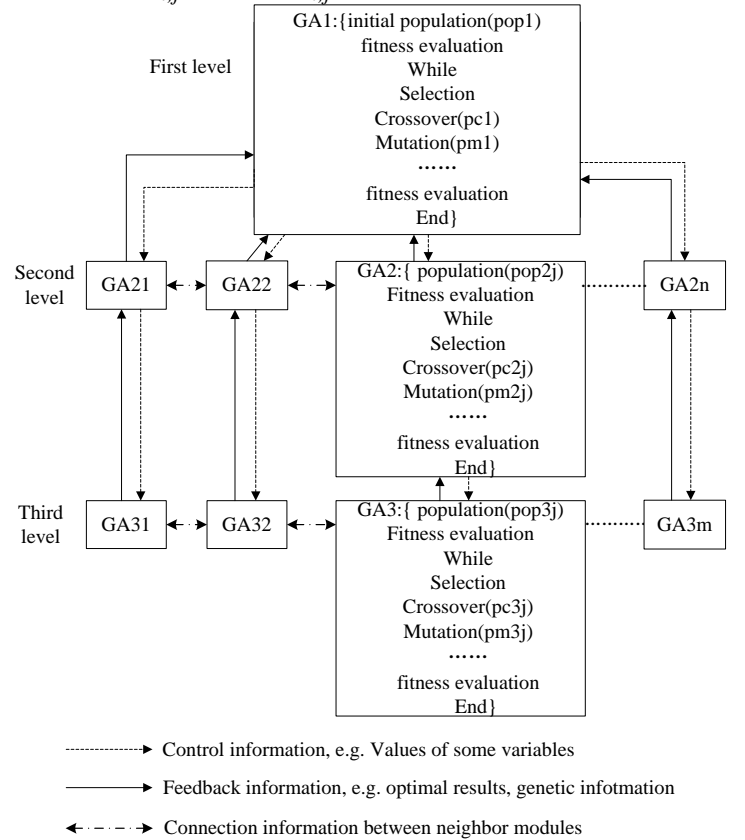

Fig. 2. Block diagram of MLGA

The implementation process of MLGA is as Fig. 3.

\section{Application of MultileVel Optimization Using MLGA}

In order to verify the proposed methods, an SPMSM controlled by FOC, rated at $950 \mathrm{~W}$ output power, $2000 \mathrm{r} / \mathrm{min}$ speed and $128 \mathrm{~V}$ line-to-line voltage, is used to verify the MLGA for multilevel optimization.

\section{A. Determination of Multilevel Optimization Model}

In the numerical example, a three level optimization model is selected, as shown in Fig. 5. Layer 1 and 2 optimize the structure of SPMSM, and the third level corresponding to the control layer. It is easily to divide the optimization model of SPMSM into two layers, that is, the structure level and the control level.

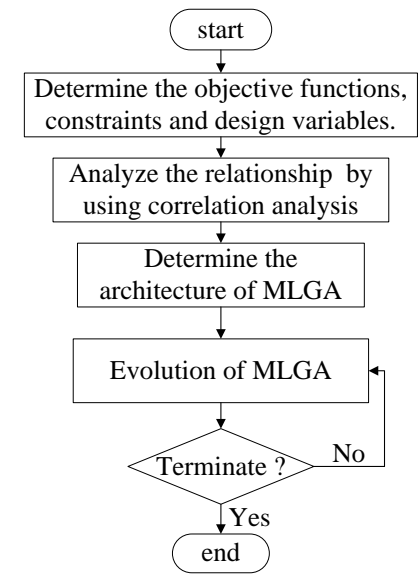

Fig. 3 Flowchart of MLGA

For the structure level of SPMSM, Correlation analysis and DOE are selected to determine the problem matrix, according to this theory, the P-values which describe the relative influence between design variables and object functions as well as constraints are analyzed by Minitab, a commercial statistic package.

The problem matrix is shown in Fig. 4.

\begin{tabular}{lcccc|} 
Variables & $h m$ & $b m$ & $N s$ & WindD \\
\cline { 3 - 5 } $\max _{1-2}(\boldsymbol{X})$ & 0.270 & 0.666 & 0.001 & 0.000 \\
$P_{2}>945 \mathrm{~W}$ & 0.005 & 0.25 & 0.32 & 0.005 \\
$S f<78 \%$ & 1.000 & 1.000 & 0.000 & 0.000 \\
\cline { 2 - 5 } & &
\end{tabular}

Fig. 4. Problem matrix of MLGA for SPMSM

In Fig. 4, the P-values of $N s$ and WindD are less than those of $\mathrm{hm}$ and $\mathrm{bm}$ with respect to objective function. That is, Ns and WindD have important influences on efficiency and costs. Therefore, $\mathrm{hm}$ and $\mathrm{bm}$ are regarded as the variables of Level 1 and $N s$ and WindD are assigned to Level 2.

\section{B. Multilevel Optimization Model}

In the level 1 and level 2, the structure of the SPMSM, and in this model, the stator and rotor cores are not permitted to be modified due to manufacture limitation. That is, the coil pitch, parallel branches and wires per conductor of 3-phase windings are fixed. The magnet thickness and width, the diameter of conductor and the conductors per slot are chosen as design variables.

The optimization objective to level 1 and level 2 are to achieve maximum of efficiency with reasonable cost of conductors and magnets. The constraints are fill factor and rated output power. The optimization model of level 1 and level 2 can be described as (4).

$$
\begin{aligned}
& \max f_{1-2}\left(X_{1}\right)=K /\left(\omega_{1} \frac{\cos t(\mathrm{Cu})}{\max (\mathrm{Cu})}+\omega_{2} \frac{\cos t(\mathrm{PM})}{\max (\mathrm{PM})}+\omega_{3} \frac{100-\eta}{100}\right) \\
& \text { s.t. } s f<0.78 \\
& \quad p_{2}>745 \mathrm{~W}
\end{aligned}
$$

where, design variable $\boldsymbol{X}_{I}=[\mathrm{hm} b m$ Ns WindD $] ; h m$ and $b m$ are the magnet thickness and width. Ns and windD are the conductors per slot and the conductor diameter, which are all discrete variables. $\operatorname{Max}(\mathrm{Cu})$ and $\operatorname{Max}(\mathrm{PM})$ are possible maximum of the cost of stator windings and permanent magnets, respectively; $\operatorname{Cost}(\mathrm{Cu})$ and $\operatorname{Cost}(\mathrm{PM})$ represent the cost of stator windings and magnets, respectively; $\eta$ is the 
efficiency of SPMSM, $K, \omega_{1}, \omega_{2}$ and $\omega_{3}$ are weight factors defined by designer. $P_{2}$ is output power and $s f$ is fill factor.

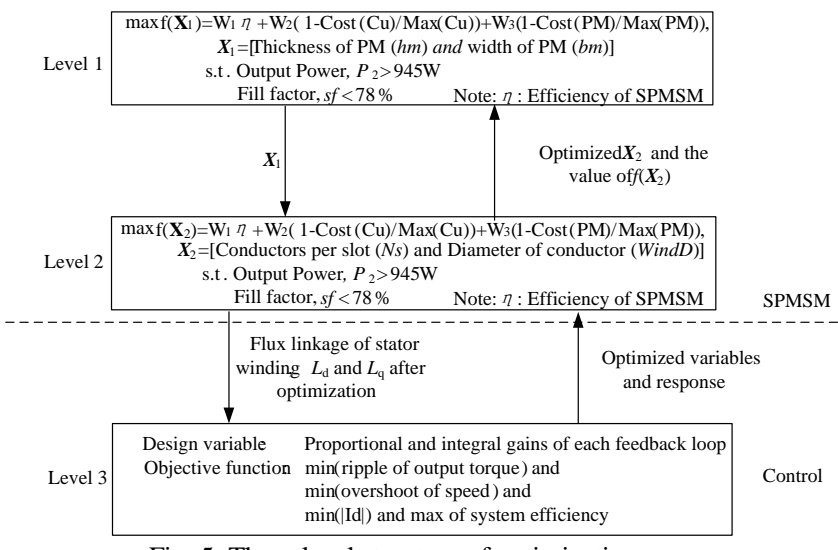

Fig. 5. Three-level structure of optimization

In the level 3, FOC was selected as the control strategy in this paper, it is known that FOC machines need two constants as in put reference: the torque component (aligned with the $\mathrm{q}$ co-ordinate) and, the flux component (aligned with the $\mathrm{d}$ coordinate). For the direct component of stator current only serves to produce waste heat and aggravate bearing wear, so the flux component corresponding to the $d$ co-ordinate is set as zero in order to minimize the direct component of stator current. The referenced speed is set as rated $2000 \mathrm{rpm}$. The block diagram of SPMSM motor drive is shown in Fig. 4. Three PI controller are used for current ( $d$ co-ordinate and $q$ co-ordinate component respectively) and speed control, Integral gain factor and proportional gain factor of PI controller to $d$ co-ordinate and $q$ co-ordinate component of stator current and speed are chosen as design variables, account to six variables. In the control layer the objective is to minimal the ripple of output torque and the overshoot of rotor speed minimal direct component of stator current Id in order to maximum the system efficient, and the objective can be formula as (5):

$\min f_{3}\left(X_{2}\right)=\alpha_{1} \cdot$ Tqripple $+\alpha_{2} \cdot$ Ovshtspd $+\alpha_{3} \cdot I_{d}$

s.t. Tqripple $\leq 0.5$

$$
\begin{aligned}
& \text { Ovshtspd } \leq 0.5 \% \\
& I_{d} \leq 0.45 \mathrm{~A}
\end{aligned}
$$

where $\boldsymbol{X}_{2}$ is design variables, Tqripple output of the torque ripple, Ovshtspd is overshoot of speed, $\mathrm{I}_{\mathrm{d}}$ is direct component of stator current, $a_{1}, a_{2}, a_{3}$ are weight factors same as (4). It is worth mentioning that the control layer is implemented with the response optimization toolbox in MATLAB. Fig. 6 describes the FOC vector control block diagram used in the control layer of SPMSM, The optimization process of level three is terminated when all the constraints are met.

The design variable $\boldsymbol{X}_{1}$ and $\boldsymbol{X}_{2}$ are set of mixed-discrete variables and $f_{1-2}\left(\boldsymbol{X}_{1}\right)$ and $f_{3}\left(\boldsymbol{X}_{2}\right)$ are multi-modal objective functions.

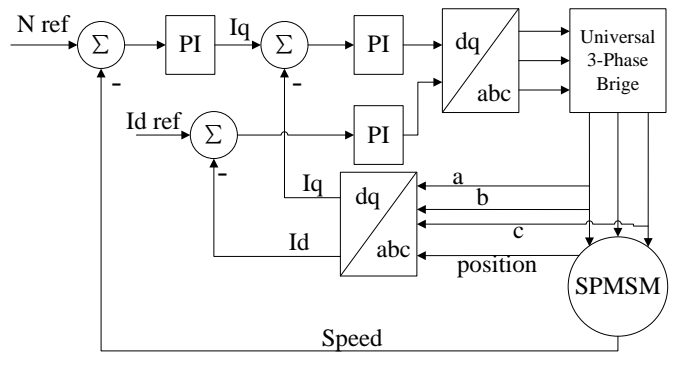

Fig. 6 FOC control block diagram

\section{FEM for no-load EMF and $L_{a d}$ and $L_{a q}$}

On Level 1, considering the nonlinear characteristics of the core, the static FEM is applied to calculate the no-load EMF per turn and the d- and q-axis components of inductances, i.e. $L_{a d}$ and $L_{a q}$, per turn to acquire the high accurate parameters when the magnet thickness and width are changed. Before solving $L_{a d}$ and $L_{a q}$, the nonlinear FEM should be conducted excited by permanent magnets only and the permeability of each finite element needs to be saved. When linear FEM is applied to calculate $L_{a d}$ and $L_{a q}$, the saved permeability will be assigned to corresponding elements. Fig. 7 pictures the magnetic field distribution when $L_{a q}$ is calculated. Fig. 5 shows the three-level architecture of optimization for SPMSM.

\section{Dynamic Adjustment of GA Operator}

In order to overcome the optimization process converged to the local optimal, on each level, if the fitness maintains in a defined interval during several consecutive generations, the mutation operator $P_{m u}$ is automatically adjusted according to (6)

$$
P_{m u}= \begin{cases}P_{m} & , n_{\text {unchange }}=0 \\ \frac{n_{\text {unchange }}}{\text { maxgeneration }} \omega & , n_{\text {unchange }}>0\end{cases}
$$

where $P_{m u}$ is dynamic mutation value, $P_{m}$ is initial mutation value, $n_{\text {unchange }}$ is the number of unchanged consecutive generations of population fitness, $\omega$ is the regulator and maxgeneration is the terminating iteration.

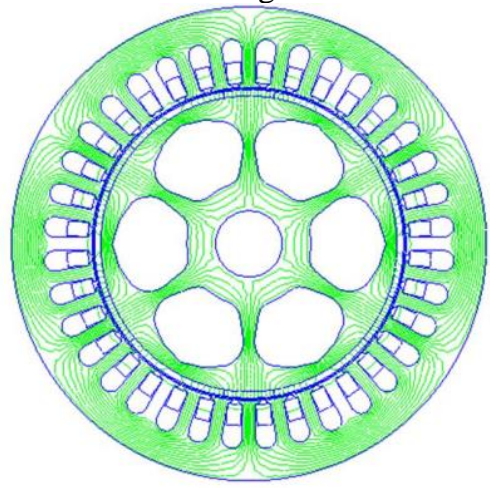

Fig. 7 Magnetic field distribution when $L_{a q}$ is calculated.

\section{E. Numerical results}

The design variables, optimal results and comparison of MLGA and traditional GA on Level 1 and 2 are listed in TABLE I. 
The proportional and integral gains calculated on the third level are listed in Table. 2. Fig. 8 illustrates the speed of SPMSM before and after PI controller parameters optimization. It can be seen from Fig. 8 that the overshoot of the rotor speed not larger than $0.7 \mathrm{rpm}$. Fig. 9 shows that the efficiency increased about $2.5 \%$, and the output electromagnetic torque are more smooth and the ripple is lower as shown in Fig. 10. From Fig. 11 we find that the $d$ coordinate component of stator current if decreased to nearly zero rapidly after optimization.

TABLE I

OPTIMAL RESULTS FOR SPMSM IN LEVEL 1 AND 2

\begin{tabular}{|c|c|c|c|}
\hline Variables and performances & $\begin{array}{c}\text { Original } \\
\text { design }\end{array}$ & $\begin{array}{c}\text { Multilevel } \\
\text { GA }\end{array}$ & $\begin{array}{c}\text { Traditional } \\
\text { GA }\end{array}$ \\
\hline Thickness of PM, $h_{m} / \mathrm{cm}$ & 0.18 & 0.23 & 0.21 \\
\hline Width of PM, $b_{m} / \mathrm{cm}$ & 3.14 & 3.03 & 3.03 \\
\hline Conductors per slot, $N s$ & 72 & 67 & 66 \\
\hline Diameter of conductor, WindD $/ \mathrm{mm}$ & 0.5 & 0.56 & 0.56 \\
\hline Back-EMF, $E_{0} / \mathrm{V}$ & 66.0 & 61.9 & 60.9 \\
\hline q-axis component of current, $I_{q} / \mathrm{A}$ & 4.78 & 5.27 & 5.37 \\
\hline d-axis component of current, $I_{d} / \mathrm{A}$ & 1.60 & 0.05 & 0.15 \\
\hline Efficiency, $\eta(\%)$ & 83.7 & 86.4 & 86.1 \\
\hline Cost of winding / RMB & 72.6 & 84.7 & 83.5 \\
\hline Cost of PM / RMB & 41.3 & 50.9 & 45.5 \\
\hline Output power,$P_{2} / \mathrm{W}$ & 946 & 949.5 & 951 \\
\hline Fill factor, $s f(\%)$ & 67 & 77.7 & 76.5 \\
\hline
\end{tabular}

TABLE II

OPTIMAL RESULTS FOR CONTROL IN LEVEL 3

\begin{tabular}{|l|c|c|}
\hline Variables and performances & Initial values & MLGA \\
\hline Proportional gain in speed loop & 1 & 18 \\
\hline Integral gain in speed loop & 1 & 0.2 \\
\hline Proportional gain in $I_{\mathrm{d}}$ loop & 1 & 20 \\
\hline Integral gain in $I_{\mathrm{d}}$ loop & 1 & 0.32 \\
\hline Proportional gain in $I_{\mathrm{q}}$ loop & 1 & 29 \\
\hline Integral gain in $I_{\mathrm{q}}$ loop & 1 & 2 \\
\hline
\end{tabular}

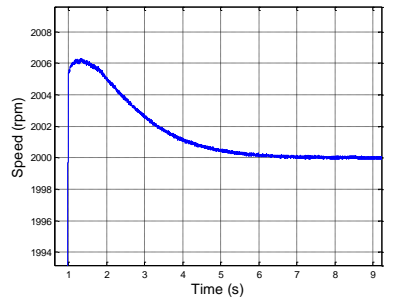

(a) Before optimization

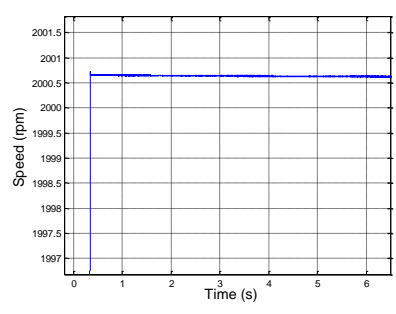

(b) After optimization
Fig. 8. Transient speed before and after optimization

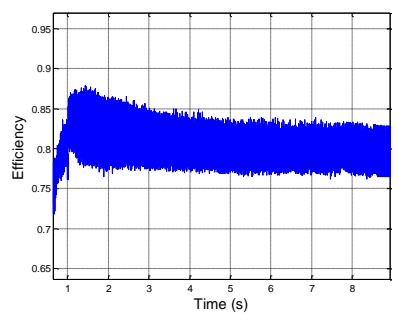

(a) Before optimization

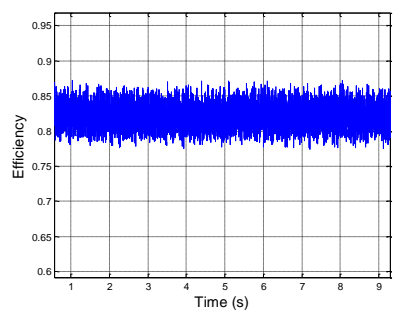

(b) After optimization
Fig. 9. Efficiency before and after optimization (a) Before optimization

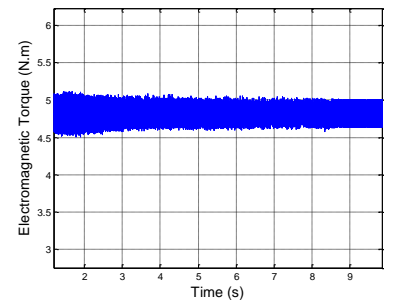

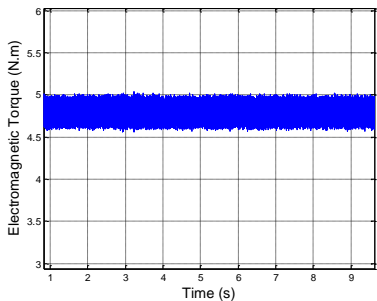

(b) After optimization

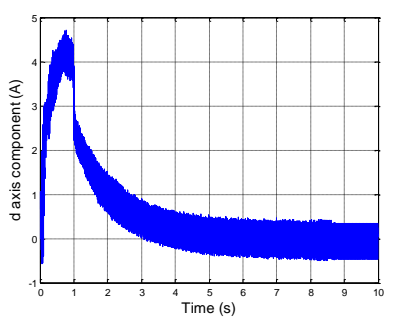

(a) Before optimization

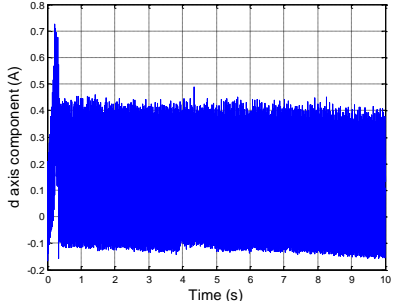

(b) After optimization
Fig. 11. $d$-axis component of stator current before and after optimization

\section{CONCLUSION}

According to the features and decision-making sequences, many real-world optimization problems in the engineering systems could be solved in multilevel procedures. This paper proposes an MLGA algorithm for SPMSM drive system to achieve complex multi-objective functions. The Correlation analysis is applied to construct the three-level structure and dynamic mutation operators on each level may dependently improve the convergence of the MLGA. It can be seen that the performances of both SPMSM and its controller can be optimized by using MLGA.

\section{REFERENCES}

[1] Barthelemy J-FM. Engineering design application of multilevel optimization methods. In Computer Aided Optimum Design of Structures, Brebbia CA, Hernandez S (eds). Springer: Berlin, 1989; 113-122.

[2] Q. S. Li, D. K. Liu, A. Y. T. Leung, N. Zhang and Q. Z. Luo. "A multilevel genetic algorithm for the optimum design of structural control systems". Int. J. Numer. Meth. Engng 2002; 55:817-834.

[3] R. Hooke and T.A. Jeeves, "Direct Search", Journal of ACM, Vol.8, 1961, pp.212-229.

[4] Shuhong Wang, Jie Qiu, Qingfu Li, Jian Guo Zhu and Semyung Wang, "Application of Petri Net in Development of Finite Element Analysis Package for Electromagnetic Fields", IEEE Transactions on Magnetics, Vol. 42, No. 4, 2006, 1255 - 1258.

[5] O.A. Mohammed, D.C. Park and F.G. Uler, "Design Optimisation of Electromagnetic Devices using Artificial Neural Networks", the Second International Forum on Applications of Neural Networks to Power Systems, 19-22 April 1993, pp.361-364

[6] Shuhong Wang, Xiangjun Meng, Jie Qiu,et al. Multilevel Optimization for Surface Mounted PM Machine Incorporating With FEM. IEEE Trans. Magn. vol 45. No 10 2009, pp.4700-4703 